\title{
On Determining the Optimal Controls and Trajectories of Continuous-Time Linear-Quadratic Regulator Problems Using Iterative Numerical Technique - I
}

\author{
F. M. ADERIBIGBE, K. J. ADEBAYO and O. S. ADETOLAJU \\ Department of Mathematical Sciences, Ekiti State University, Ado Ekiti, Nigeria.
}

\begin{abstract}
This paper investigates the application of the iterative Steepest Descent Method (SDM) to the solution of nonlinear two-point boundary-value problems for the optimal controls and trajectories of continuous-time linear-quadratic regulator problems. Numerical results show some improvement over the classical methods.
\end{abstract}

Keywords: Optimal Control, Continuous Linear Regulator, Steepest Descent Method.

\section{Introduction}

The variational approach to solving optimal control problems leads to a nonlinear two point boundaryvalue problems that cannot be solved analytically to obtain the optimal control law, or even an optimal open loop control. In problems with plant dynamics and quadratic performance criteria (linear regulator and tracking systems), it has been found that, it is possible to obtain the optimal control law by numerically integrating a matrix differential equation of the Riccati type.

\section{Continuous-Time Linear-Quadratic Regulator Problems}

In this section we shall consider an important class of optimal control problems. The process to be controlled is described by the state equations:

$\dot{x}(t)=A(t) x(t)+B(t) u(t), \quad x\left(t_{0}\right)=x_{0}$,

$y(t)=C(t) x(t)$

where $x(t)$ is an $n$-dimensional state vector, $u(t)$ is the $m$-dimensional plant control input vector and $y(t)$ is an $r$-dimensional output vector, with $(0 \leq r \leq m \leq n)$; and $A(t), B(t)$ and $C(t)$ are $n \times n, n \times m$ and $r \times n$ matrices, respectively.

According to [4], the plant described above is a continuous-time, linear dynamical system with the following properties:

1. A time set $\left\{t_{k}\right\}$ such that $\left\{t_{i}\right\}=(-\infty, \infty)=E_{1}$ for $T>t_{0}$, where $t_{0}$ is the initial time and $\mathrm{T}$ is the final or terminal time.

2. A set of states $\left\{x_{i}(t)\right\}=X=E_{n}$, called the state space, where $E_{n}$ is the $n$-dimensional Euclidean vector space.

3. A set of inputs or controls $\left\{u_{j}(t)\right\}=U=E_{m}$, called the input or control space, where $E_{m}$ is the $m$-dimensional Euclidean vector space.

4. A function space $\Omega$ whose elements are bounded, measurable functions, which map $E_{1}$ into $\mathrm{U}$.

5. A set of outputs $\{y\}=Y=E_{r}$, called the output space.

If the control $u($.$) is a given element of \Omega$, let $x_{u}=\varphi\left(t ; t_{0}, x_{0}, u().\right)$ denote the solution of the system (2.1) starting from $x_{0}$ at time $t_{0}$ [that is , $x\left(t_{0}\right)=x_{0}$ ], and generated by the control $u($.$) . Furthermore, let$ $y_{u}(t)=C(t) x_{u}(t)$ be the corresponding output trajectory. Then, the optimal linear regulator problem is to determine the control $u($.$) , which, as we shall see below, minimizes a quadratic performance index. More$ specifically, an explicit solution of (2.1), given an initial state, $x_{0}$ at $t_{0}$ and $t \geq t_{0}$, is as follows: (see [3])

$x(t)=\Phi\left(t, t_{0}\right) x_{0}+\int_{t_{0}}^{t} \Phi(t, \tau) B(\tau) u(\tau) d \tau$

where $\Phi(t, \tau)=e^{A(t-\tau)}$ is the state transition matrix associated with the constant matrix $A(t)$. Similarly, the solution to (2.2), given an initial state, $x_{0}$ and input, $\mathrm{u}(\mathrm{t})$ on $\left[t_{0}, \infty\right)$, is (see [1]):

$x(t)=C(t) \Phi\left(t, t_{0}\right) x_{0}+\int_{t_{0}}^{t} \mathrm{G}(t, \tau) u(\tau) d \tau$

where

$G(t, \tau)=\left\{\begin{array}{ll}C(t) \Phi\left(t, t_{0}\right) B(\tau) ; & \text { for } t \geq \tau \\ 0 \quad & \text { for } t<\tau\end{array}\right\}$

is the impulse response matrix. 
Therefore, and as mentioned above, the optimal linear regulator problem for a linear dynamic system entails the determination of the optimal control $u^{*}(t), t \in\left[t_{0}, T\right]$, which minimizes the quadratic performance index: (see [1] and [4])

$J\left(x, t_{0}, T, u().\right)=\frac{1}{2} x^{T}(T) H x(T)+\frac{1}{2} \int_{t_{0}}^{T}\left\{x^{T}(t) Q(t) x(t)+u^{T}(t) R(t) u(t)\right\} d t$

where $H$ is a real symmetric positive semi definite (nonzero) $n \times n$ matrix; the "terminal state" $x_{u}(T) \in X$ is unconstrained, and the terminal time, $T$, may be either fixed prior or unspecified $\left(T>t_{0}\right)$. The superscript $T$ denotes matrix transposition. $Q(t)$ is a real symmetric $n \times n$ positive definite matrix. $\mathrm{H}$ and $Q(t)$ are not both identically zero. Since $R^{-1}(t)$ is positive definite, it possesses a unique positive definite square root, $R^{-1 / 2}(t)$. Similarly, the positive semi definiteness of $Q(t)$ implies the existence of the unique positive semi definite square root $Q^{-1 / 2}(t)$. The notation $H \geq 0$ will be used to indicate that, the matrix $H$ is positive semi definite, that is, $x^{T} H x \geq 0$. Similarly, the notation $\mathrm{H}>0$ will be used to indicate that $\mathrm{H}$ is positive definite. In order to minimize the performance index $J$, it is necessary that $J$ is finite, which means that it will become infinite if uncontrollable. The weighting matrices $Q(t)$ and $R(t)$ are selected by the control system designer to place bounds on the trajectory and control, respectively, while the matrix $\mathrm{H}$ and the terminal penalty $\operatorname{cost} x^{T}(T) H x(T)$ are included in order to ensure that $x(t)$ stays close to zero near the terminal time. From a design point of view, the control system designer may design the system so that the term $x^{T} Q(t) x(t)^{*}$ is chosen to penalize deviations of the regulated state $x(t)$ from the desired equilibrium condition $x(t)=0$, where as the term $u^{T} R(t) u(t)$ discourages the use of excessively large control effort.

\section{The Steepest Descent Method (SDM)}

Our discussion of the SDM (a gradient method) will begin by considering an analogous calculus problem. Let $f$ be a function of two independent variables $y_{1}$ and $y_{2}$; the value of the function $f$ at the point $y_{1}, y_{2}$ is denoted by $f\left(y_{1}, y_{2}\right)$. It is desired to find the point $y_{1}^{*}, y_{2}^{*}$, where $f$ assumes its minimum values, $f\left(y_{1}^{*}, y_{2}^{*}\right)$. According to [6], if it is assumed that the variables $y_{1}$ and $y_{2}$ are not constrained by any boundaries, a necessary condition for $y_{1}^{*}, y_{2}^{*}$ to be a point where $f$ has a (relative) minimum is that, the differential of $f$ vanish at $y_{1}^{*}, y_{2}^{*}$, that is,

$$
\begin{aligned}
d f\left(y_{1}^{*}, y_{2}^{*}\right) & =\left[\frac{\partial f}{\partial y_{1}}\left(y_{1}^{*}, y_{2}^{*}\right)\right] \Delta y_{1}+\left[\frac{\partial f}{\partial y_{2}}\left(y_{1}^{*}, y_{2}^{*}\right)\right] \Delta y_{2} \\
& \triangleq\left[\frac{\partial f}{\partial y}\left(y^{*}\right)\right]^{T} \Delta y=0
\end{aligned}
$$

$\frac{\partial f}{\partial y}$ is called the gradient of $f$ with respect to $y$. Since $y_{1}$ and $y_{2}$ are independent, the components of $\Delta y$ are independent and (3.1) implies, that is,

$$
\frac{\partial f}{\partial y}\left(y^{*}\right)=0 \text {. }
$$

However, for $f\left(y^{*}\right)$ to be a relative minimum, it is necessary that the gradient of $f$ be zero at the point $y^{*}$. Equation (3.2) represents two algebraic equations that are generally nonlinear which, if the algebraic equations cannot be determined analytically for $y^{*}$, one possible approach is to visualize the minimization as a problem in hill climbing. As in [6], it is opined that, the function $f$ defines hills and valleys in the three-dimensional $\left(y_{1}, y_{2}, f\left(y_{1}, y_{2}\right)\right)$ space. One way to find the bottom of a valley is to pick a trial point $y^{(0)}$ and climb in a downward direction until a point $y^{*}$ is reached where further movement in any direction increases the function value. To make the climbing procedure efficient, we chose to climb in the direction of steepest descent, thus ensuring that the shortest distance is traveled in reaching the bottom of the hill. The direction of the steepest descent at $y^{(0)}$ is determined by evaluating the slope or gradient of the hill at the point $y^{(0)}$. It is evident that, the gradient vector is normal to the elevation contour and $z\left(y^{(0)}\right)$ is the unit vector in the gradient direction at the point $z\left(y^{(0)}\right)$; that is,

$z\left(y^{(0)}\right) \triangleq \frac{\frac{\partial f}{\partial y}\left(y^{(0)}\right)}{\left\|\frac{\partial f}{\partial y}\left(y^{(0)}\right)\right\|}=\frac{\frac{\partial f}{\partial y}\left(y^{(0)}\right)}{\sqrt{\left[\frac{\partial f}{\partial y_{1}}\left(y^{(0)}\right)\right]^{2}+\left[\frac{\partial f}{\partial y_{2}}\left(y^{(0)}\right)\right]^{2}}}$.

Climbing in the direction of the vector $-z\left(y^{(0)}\right)$, the change in $y$ is given by

$\Delta y \triangleq y^{(1)}-y^{(0)}=-\tau z\left(y^{(0)}\right)$,

where $\tau>0$ is the step length. With this selection for $\Delta y$, the differential, which is a linear approximation of the change in $f$, becomes

$d f\left(y^{(0)}\right)=-\tau\left[\frac{\partial f}{\partial y}\left(y^{(0)}\right)\right]^{T} z\left(y^{(0)}\right)$

or, by using (3.3), 


$$
\begin{aligned}
d f\left(y^{(0)}\right) & =\frac{-\tau\left\{\left[\frac{\partial f}{\partial y_{1}}\left(y^{(0)}\right)\right]^{2}+\left[\frac{\partial f}{\partial y_{2}}\left(y^{(0)}\right)\right]^{2}\right\}}{\left\|\frac{\partial f}{\partial y}\left(y^{(0)}\right)\right\|} \\
& =-\tau \sqrt{\left[\frac{\partial f}{\partial y_{1}}\left(y^{(0)}\right)\right]^{2}+\left[\frac{\partial f}{\partial y_{2}}\left(y^{(0)}\right)\right]^{2}} .
\end{aligned}
$$

Notice that this implies that

$$
d f\left(y^{(0)}\right) \leq 0,
$$

With the equality holding if and only if $\frac{\partial f}{\partial y}$ is zero at $y^{(0)}$. This iterative procedure is continued by calculating $z\left(y^{(1)}\right)$, the unit vector in the gradient direction at $y^{(1)}$, and using the generalization of (3.4) to determine the next point, $y^{(2)}$.

$\Delta y \triangleq y^{(i+1)}-y^{(i)}=-\tau z\left(y^{(i)}\right)$

It must be stated that, a suitable value for the step length, $\tau$, must also be selected. If the value of $\tau$ is too large, definitely, we will overshoot the mark and if $\tau$ is too small, too much time is being spent trying to measure the slopes and not enough time is spent climbing.

\section{Minimization of Functionalsby SDM}

Suppose that a nominal control history $u^{(i)}(t), t \in\left[t_{0}, t_{f}\right]$, is known and used to solve the differential equations

$\dot{x}^{(i)}(t)=a\left(x^{(i)}(t), u^{(i)}(t), t\right)$

$\dot{p}^{(i)}(t)=-\frac{\partial \mathcal{H}}{\partial x}\left(x^{(i)}(t), u^{(i)}(t), p^{(i)}(t), t\right)$

so that the nominal state-costate trajectory $x^{(i)}, p^{(i)}$ satisfies the boundary conditions

$x^{(i)}\left(t_{0}\right)=x_{0}$

$p^{(i)}\left(t_{f}\right)=\frac{\partial h}{\partial x}\left(x^{(i)}\left(t_{f}\right)\right)$.

If this nominal control history also satisfies

$\frac{\partial \mathcal{H}}{\partial u}\left(x^{(i)}(t), u^{(i)}(t), p^{(i)}(t), t\right)=0, \quad t \in\left[t_{0}, t_{f}\right]$,

Then $x^{(i)}(t), u^{(i)}(t)$ and $p^{(i)}(t)$ are extremal. Suppose that (4.5) is not satisfied; the variation of the augmented functional $J_{a}$ on the nominal state-costate-control history is

$$
\begin{aligned}
\delta J_{a}= & {\left[\frac{\partial h}{\partial x}\left(x^{(i)}\left(t_{f}\right)\right)-p^{(i)}\left(t_{f}\right)\right]^{T} \delta x\left(t_{f}\right)+\int_{t_{0}}^{t_{f}}\left\{\left[\dot{p}^{(i)}(t)+\frac{\partial \mathcal{H}}{\partial x}\left(x^{(i)}(t), u^{(i)}(t), p^{(i)}(t), t\right)\right]^{T} \delta x(t)\right.} \\
& \left.+\left[\frac{\partial \mathcal{H}}{\partial u}\left(x^{(i)}(t), u^{(i)}(t), p^{(i)}(t), t\right)\right]^{T} \delta u(t)+\left[a\left(x^{(i)}(t), u^{(i)}(t), t\right)-\dot{x}^{(i)}(t)\right]^{T} \delta p(t)\right\} d t,
\end{aligned}
$$

where $\delta x(t) \triangleq x^{(i+1)}(t)-x^{(i)}(t), \delta u(t) \triangleq u^{(i+1)}(t)-u^{(i)}(t)$, and

$\delta p(t) \triangleq p^{(i+1)}(t)-p^{(i)}(t)$.

If (4.1) through (4.4) are satisfied, then

$\delta J_{a}=\int_{t_{0}}^{t_{f}}\left\{\left[\frac{\partial \mathcal{H}}{\partial u}\left(x^{(i)}(t), u^{(i)}(t), p^{(i)}(t), t\right)\right]^{T} \delta u(t) d t\right.$

Recalling that $\delta J_{a}$ is the linear part of the increment $\Delta J_{a} \triangleq J_{a}\left(u^{(i+1)}\right)-J_{a}\left(u^{(i)}\right)$, and that if the norm of $\delta u,\left\|u^{(i+1)}(t)-u^{(i)}(t)\right\|$, is small, the sign of $\Delta J_{a}$ will be determined by the sign of $\delta J_{a}$. Since our goal is to minimize $J_{a}$, we wish to make $\Delta J_{a}$ negative. If we select the change in $u$ as

$$
\delta u(t)=u^{(i+1)}(t)-u^{(i)}(t)=-\tau \frac{\partial \mathcal{H}}{\partial x}^{(i)}(\mathrm{t}), \quad t \in\left[t_{0}, t_{f}\right],
$$

with $\tau>0$, then

$\delta J_{a}=-\tau \int_{t_{0}}^{t_{f}}\left[{\frac{\partial \mathcal{H}^{(i)}}{\partial u}}^{(i)}(\mathrm{t})\right]^{T}\left[{\frac{\partial \mathcal{H}^{(i)}}{\partial u}}^{(\mathrm{t})}\right] d t \leq 0$,

Because the integrand is nonnegative for all $t \in\left[t_{0}, t_{f}\right]$. The equality holds if and only if

${\frac{\partial \mathcal{H}^{(i)}}{\partial u}}^{(\mathrm{t})}=0$ for all $t \in\left[t_{0}, t_{f}\right]$.

Selecting $\delta u$ in this manner, with $\|\delta u\|$ sufficiently small, ensures that each value of the performance measure will be at least as small as the preceding value. Eventually, when $J_{a}$ reaches a relative minimum, the vector $\frac{\partial \mathcal{H}}{\partial u}$ will be zero throughout the time interval $\left[t_{0}, t_{f}\right]$. Subject to these, we have assumed that (4.1) to (4.4) are satisfied. On how this is achieved, we outline the algorithm as it would be executed using a digital computer. 


\section{The Steepest Descent Algorithm}

According to [6], the optimal control problem (2.1) and (2.6) may be solved with the SDM via the following steps:

a. Select a discrete approximation to the nominal control history $u^{(0)}(t), t \in\left[t_{0}, t_{f}\right]$, and store this in the memory of the digital computer. This is done by subdividing the interval $\left[t_{0}, t_{f}\right]$ into $\mathrm{N}$ subintervals (generally of equal duration) and considering the control $u^{(0)}$ as being piecewise- constant during each of these subintervals; that is

$u^{(0)}(t)=u^{(0)}\left(t_{k}\right), t \in\left[t_{k}, t_{k+1}\right), k=0,1,2, \ldots, N-1$.

Let the iteration index $i$ be zero.

b. Using the nominal control history $u^{(i)}$, integrate the state equations from $t_{0}$ to $t_{f}$ with initial conditions $x\left(t_{0}\right)=x_{0}$ and store the resulting state trajectory $x^{(i)}$ as a piecewise-constant vector function.

c. Calculate $p^{(i)}\left(t_{f}\right)$ and substitute $x^{(i)}\left(t_{f}\right)$ from step (b) intoequation (4.4). Using this value of $p^{(i)}\left(t_{f}\right)$ as the initial condition and the piecewise-constant values of $x^{(i)}$ stored in step (b), integrate the costate equations from $t_{f}$ to $t_{0}$, evaluating

$\frac{\partial \mathcal{H}^{(i)}(t)}{\partial u}, t \in\left[t_{0}, t_{f}\right]$, and store this function in piecewise-constant fashion.

d. If $\gamma$,is a preselected positive constant such that

$\left\|\frac{\partial \mathcal{H}^{(i)}}{\partial u}\right\| \leq \gamma$,

and

$\left\|\frac{\partial \mathcal{H}^{(i)}}{\partial u}\right\|^{2} \triangleq \int_{t_{0}}^{t_{f}}\left[\frac{\partial \mathcal{H}^{(i)}}{\partial u}(t)\right]^{T}\left[\frac{\partial \mathcal{H}^{(i)}}{\partial u}(t)\right] d t$,

terminate the iterative procedure, and output the extremal state and control. If the stopping criterion (5.2) is not satisfied, then, generate a new piecewise-constant function given by

$u^{(i+1)}\left(t_{k}\right)=u^{(i)}\left(t_{k}\right)-\tau \frac{\partial \mathcal{H}^{(i)}}{\partial u}\left(t_{k}\right), k=0,1, \ldots, N-1$,

where $u^{(i)}(t)=u^{(i)}\left(t_{k}\right)$ for $t \in\left[t_{k}, t_{k+1}\right), k=0,1, \ldots, N-1$.

Then, replace $u^{(i)}\left(t_{k}\right)$ by $u^{(i+1)}\left(t_{k}\right), k=0,1, \ldots, N-1$, and return to step (b) above.

The value of the terminating constant $\gamma$, will depend on the problem to be solved and the accuracy desired of the solution. The step length $\tau$, is generally determined by some ad- hoc strategy. One possible strategy is to select a value $\tau$ which attempts to affect a certain value of $\Delta J_{a}$.

From (5.4) we observe that

$\delta J_{a}=-\tau\left\|\frac{\partial \mathcal{H}^{(i)}}{\partial u}\right\|^{2} \leq 0$.

To effect an approximate change of $q$ percent in $J_{a}$, select $\tau$ as

$\tau=\frac{\frac{q}{100}|J a|}{\left\|\frac{\partial \mathcal{F}^{(i)}}{\partial u}\right\|^{2}}$.

An alternative strategy for selecting $\tau$ is to use a single variable search. We choose an arbitrary starting value of $\tau$, compute $\frac{\partial \mathcal{H}^{(i)}}{\partial u}$, and evaluate $u^{(i+1)}$ using (4.5).

In summary, we now present the SDM for solving continuous-time linear-quadratic regulator problems as follows in the next section.

\section{Computational Results}

The following linear regulator problems were solved using the SDM algorithm described above.

\section{Problem (P1)}

What is the optimal trajectory and control for the system

$\dot{x}(t)=-3 x(t)+2 u(t) ; 0 \leq t \leq 1$,

$x(0)=2$ and $u_{0}(t)=1.0$, that minimizes the performance measure

$\mathrm{J}=x^{2}(1)+\frac{1}{2} \int_{0}^{1}\left\{x^{2}(t)+u^{2}(t)\right\} d t$

Problem (P2)

Find the optimal trajectory and control for the system

$\dot{x}(t)=-x(t)+u(t) ; 0 \leq t \leq 1$,

$x(0)=4$ and $u_{0}(t)=1.0$, that minimizes the performance measure

$\mathrm{J}=x^{2}(1)+\int_{0}^{1} \frac{1}{2} u^{2}(t) d t$ 


\section{Problem (P3)}

Minimize $\mathrm{J}=x^{2}(1)+\frac{1}{2} \int_{0}^{1}\left[4 x^{2}(t)+2 u^{2}(t)\right] d t$

Subject to the constraint

$\dot{x}(t)=-x(t)+u(t) ; 0 \leq t \leq 1, x(0)=3$ and $u_{0}(t)=1.0$.

\section{Problem (P4)}

Minimize $\mathbf{J}=\frac{1}{2} x^{2}(0.04)+\frac{1}{4} \int_{0}^{0.04}\left[x^{2}(t)+u^{2}(t)\right] d t$

Subject to the constraint

$\dot{x}(t)=-10 x(t)+u(t) ; 0 \leq t \leq 0.04, x(0)=2$ and $u_{0}(t)=1.0$.

\section{Problem (P5)}

Minimize $\mathbf{J}=10 x_{1}^{2}(T)+\frac{1}{2} \int_{0}^{T}\left[x_{1}^{2}(t)+2 x_{2}^{2}(t)+u^{2}(t)\right] d t$ $\dot{x}(t)=\left(\begin{array}{rr}0 & 1 \\ -1 & -2\end{array}\right)\left(\begin{array}{l}x_{1} \\ x_{2}\end{array}\right)+\left(\begin{array}{l}0 \\ 1\end{array}\right) u, x(0)=\left(\begin{array}{r}1 \\ -1\end{array}\right)$ and $u_{0}(t)=\left(\begin{array}{l}0 \\ 1\end{array}\right)$. The final time $\mathrm{T}=10$.

The computational results of the above problems are presented in Tables $1-5$.

\section{Conclusion}

Computationally, the SDM was tested on a number of continuous-time linear-quadratic regulator problems with the results obtained in each case. Our numerical results for these problems are presented in the Tables 1-4. From the tables, it can be seen that (P1) readily converged at the seventh iteration when compared with the analytical result, $x^{*}=0.00017736422$ and $u^{*}=0.00019165842$. (P2) and (P3) converged at the forth and third iterations comparing them with the analytical results $x^{*}=-0.14998465235$,

$u^{*}=-0.2735776132$ and $x^{*}=-0.3237098216, u^{*}=-0.3742215284$ respectively. While (P4) converged at the $18^{\text {th }}$ iteration to $x^{*}=0.008850547533$ and $u^{*}=0.04615397268$. In addition, the terminating criterion was fixed at $\left\|\frac{\partial \mathcal{H}^{(i)}}{\partial u}\right\| \leq 10^{-10}$. The terminating criterion is relaxed so that we could substantiate usage of the SDM in solving this class of problem. Based on the results, it is obvious that, on determining the optimal controls and trajectories of continuous-time linear-quadratic regulator problems using iterative numerical techniques, the Steepest Descent Method is relevant and recommended for use.

\section{References}

[1] Athans, M. and Falb, P. L., (1966), Optimal Control: An Introduction to the Theory and Its Applications, McGraw-Hill, New York. [2] Bryson, A. E. Jr., and W. F., Denham, (1964), “Optimal Programming Problems with Inequality Constraints II: Solution by Steepest Ascent," AIAA Journal, 25-34

[3] Burghes, D. N. and Graham, A., (1980), Introduction To Control Theory, Including Optimal Control, John Wiley \& Sons

[4] George M. Siouris, (1996), An Engineering Approach To Optimal Control And Estimation Theory, John Wiley \& Sons, Inc.

[5] Kelly, H. J., (1962), "Method of Gradient," Optimization Techniques with Applications To Aerospace Systems. G. Leitmann, Ed. New York: Academic Press Inc.

[6] Kirk, E. Donald, (2004), Optimal control theory: An introduction I, Englewood Cliffs, N. J., Prentice-Hall,

Table 1: Computational Results for Solution of Problem (P1)

\begin{tabular}{|l|l|l|l|l|}
\hline Iteration & $\mathrm{X}(\mathrm{t})$ & $\mathrm{U}(\mathrm{t})$ & $\mathrm{P}(\mathrm{t})$ & $\frac{\partial \mathcal{H}}{\partial u}$ \\
\hline 0 & 2 & 1.0 & - & - \\
\hline 1 & 0.73304942 & 0.31356046 & 2.9321977 & 6.8643954 \\
\hline 2 & 0.23512918 & $0.94101067 \mathrm{e}-1$ & 0.94051674 & 2.19459394 \\
\hline 3 & $0.71317093 \mathrm{e}-1$ & $0.27637286 \mathrm{e}-1$ & 0.28526837 & 0.66463781 \\
\hline 4 & 0.02105820 & $0.80269919 \mathrm{e}-2$ & $0.84232826 \mathrm{e}-1$ & 0.19610294 \\
\hline 5 & $0.61333273 \mathrm{e}-2$ & $0.23176308 \mathrm{e}-2$ & 0.02453330 & $0.57093611 \mathrm{e}-1$ \\
\hline 6 & $0.17735222 \mathrm{e}-2$ & $0.66704994 \mathrm{e}-3$ & $0.70940889 \mathrm{e}-2$ & 0.01650580 \\
\hline 7 & $0.51085812 \mathrm{e}-3$ & $0.19165845 \mathrm{e}-3$ & $0.20434325 \mathrm{e}-2$ & $0.47539149 \mathrm{e}-2$ \\
\hline
\end{tabular}


On Determining The Optimal Controls And Trajectories Of Continuous-Time Linear-Quadratic

Table 2: Computational Results for Solution of Problem (P2)

\begin{tabular}{|l|l|l|l|l|}
\hline Iteration & $\mathrm{X}(\mathrm{t})$ & $\mathrm{U}(\mathrm{t})$ & $\mathrm{P}(\mathrm{t})$ & $\frac{\partial \mathcal{H}}{\partial u}$ \\
\hline 0 & 4 & 1 & - & - \\
\hline 1 & 2.10363832 & $0.58544671 \mathrm{e}-1$ & 8.41455329 & 9.41455329 \\
\hline 2 & 0.81089258 & -0.27166683 & 3.24357032 & 3.30211499 \\
\hline 3 & 0.12658452 & -0.29513395 & 0.50633809 & 0.23467126 \\
\hline 4 & -0.13999246 & -0.20962363 & -0.55996959 & -0.85510354 \\
\hline
\end{tabular}

Table 3: Computational Results for Solution of Problem (P3)
\begin{tabular}{|l|l|l|l|l|}
\hline Iteration & $\mathrm{X}(\mathrm{t})$ & $\mathrm{U}(\mathrm{t})$ & $\mathrm{P}(\mathrm{t})$ & $\frac{\partial \mathcal{H}}{\partial u}$ \\
\hline 0 & 3 & 1 & - & - \\
\hline 1 & 1.73575888 & -0.48860711 & 6.94303553 & 14.8860711 \\
\hline 2 & 0.32969141 & -0.70349952 & 1.31876564 & 2.14892418 \\
\hline 3 & -0.32340982 & -0.37442172 & -1.29363928 & -3.29077809 \\
\hline
\end{tabular}

Table 4: Computational Results for Solution of Problem (P4)

Table 4: Computational Results for Solution of Problem (P4)
\begin{tabular}{|l|l|l|l|l|}
\hline & & & & \\
Iteration & $\mathrm{X}(\mathrm{t})$ & $\mathrm{U}(\mathrm{t})$ & $\mathrm{P}(\mathrm{t})$ & \\
\hline 0 & 2 & 1 & - & - \\
\hline 1 & 1.37360809 & 0.76263919 & 2.74721618 & 2.37360809 \\
\hline 2 & 0.94589972 & 0.5917853 & 1.89179944 & 1.70853891 \\
\hline 3 & 0.65356552 & 0.46725022 & 1.30713104 & 1.24535082 \\
\hline 4 & 0.45350237 & 0.37517496 & 0.90700475 & 0.92075259 \\
\hline 5 & 0.3163605 & 0.30602141 & 0.632721 & 0.69153546 \\
\hline 6 & 0.2221517 & 0.2532041 & 0.44430339 & 0.52817311 \\
\hline 7 & 0.15726037 & 0.21215766 & 0.31452073 & 0.41046447 \\
\hline 8 & 0.11240919 & 0.17970097 & 0.22481838 & 0.32456684 \\
\hline 9 & $0.81274513 \mathrm{e}-1$ & 0.15360342 & 0.16254903 & 0.26097548 \\
\hline 10 & $0.59543933 \mathrm{e}-1$ & 0.13228869 & 0.11908787 & 0.21314736 \\
\hline 11 & $0.44274784 \mathrm{e}-1$ & 0.11463234 & $0.88549569 \mathrm{e}-1$ & 0.17656347 \\
\hline 12 & $0.33457474 \mathrm{e}-1$ & $0.99823359 \mathrm{e}-1$ & $0.66914948 \mathrm{e}-1$ & 0.14808981 \\
\hline 13 & $0.25718192 \mathrm{e}-1$ & 0.08726920 & $0.51436383 \mathrm{e}-1$ & 0.12554155 \\
\hline 14 & $0.2011651 \mathrm{e}-1$ & $0.76530632 \mathrm{e}-1$ & $0.4023302 \mathrm{e}-1$ & 0.10738571 \\
\hline 15 & $0.16007561 \mathrm{e}-1$ & $0.67276813 \mathrm{e}-1$ & $0.32015123 \mathrm{e}-1$ & $0.92538194 \mathrm{e}-1$ \\
\hline 16 & $0.12948171 \mathrm{e}-1$ & $0.59254314 \mathrm{e}-1$ & $0.25896342 \mathrm{e}-1$ & $0.80224984 \mathrm{e}-1$ \\
\hline 17 & $0.10632915 \mathrm{e}-1$ & $0.52265592 \mathrm{e}-1$ & $0.21265829 \mathrm{e}-1$ & $0.69887229 \mathrm{e}-1$ \\
\hline 18 & $0.88505475 \mathrm{e}-2$ & $0.46153978 \mathrm{e}-1$ & $0.17701095 \mathrm{e}-1$ & $0.61116139 \mathrm{e}-1$ \\
\hline
\end{tabular}

